\title{
Change Detection from Remotely Sensed Images Based on Stationary Wavelet Transform
}

\author{
Abhishek Sharma ${ }^{1}$, Tarun Gulati \\ Electronics and Communication Department, Maharishi Markandeshwar University, Mullana, Ambala, India
}

\begin{abstract}
Article Info
Article history:

Received Apr 17, 2017

Revised Jun 21, 2017

Accepted Jul 10, 2017

\section{Keyword:}

Change detection

Stationary wavelet transform

Image fusion

Log ratio

Mean ratio

Fuzzy clustering

ABSTRACT

The major issue of concern in change detection process is the accuracy of the algorithm to recover changed and unchanged pixels. The fusion rules presented in the existing methods could not integrate the features accurately which results in more number of false alarms and speckle noise in the output image. This paper proposes an algorithm which fuses two multi-temporal images through proposed set of fusion rules in stationary wavelet transform. In the first step, the source images obtained from log ratio and mean ratio operators are decomposed into three high frequency sub-bands and one low frequency sub-band by stationary wavelet transform. Then, proposed fusion rules for low and high frequency sub-bands are applied on the coefficient maps to get the fused wavelet coefficients map. The fused image is recovered by applying the inverse stationary wavelet transform (ISWT) on the fused coefficient map. Finally, the changed and unchanged areas are classified using Fuzzy c means clustering. The performance of the algorithm is calculated in terms of percentage correct classification (PCC), overall error (OE) and Kappa coefficient $\left(\mathrm{K}_{\mathrm{c}}\right)$. The qualitative and quantitative results prove that the proposed method offers least error, highest accuracy and Kappa value as compare to its preexistences.
\end{abstract}

Copyright $(2017$ Institute of Advanced Engineering and Science. All rights reserved.

Corresponding Author:

Abhishek Sharma,

Electronics and Communication Department,

Maharishi Markandeshwar University,

Mullana, Ambala, India.

Email: abhishek.kaushik1@gmail.com

\section{INTRODUCTION}

Change detection is a process in which the multi-temporal images are compared to detect the number of pixels that have undergone change with time [1]. The most basic techniques used for change detection are differencing and ratioing. In image differencing, the corresponding pixel in one image is subtracted from the second image to generate a difference image. In image ratioing, the corresponding values of pixels in multi-temporal images are divided to get the output image. Out of these techniques, ratioing techniques are more popular because of their robustness to calibration errors [2]-[3]. However, every change detection methods have its own advantages and drawbacks in terms of the false alarms generation and accuracy of the change detection. So, it is difficult to obtain all the features from one change detector. To overcome this difficulty, the output of different change detection methods can be combined together to obtain a high quality output. Such technique of integrating the different images together is called as image fusion [4]. The output of mean ratio and $\log$ ratio can be fused together through different fusion rules to produce an output which contain features of both the operators. In the literature, many image fusion methods have been presented like discrete wavelet transform (DWT) [5] and Stationary wavelet transform [6]-[7]. However, SWT is the popular techniques for image fusion because it is shift invariant which does not allow the alteration in coefficient map if there is any small mismatch between the pixels of source image [8]. 
In Stationary wavelet transform based image fusion, the source images are decomposed into four equal sized images. One image represents the low frequency sub-band containing the approximate portion of the image while the three images representing the high frequency sub-band contain information horizontal, vertical and diagonal components respectively. In the next step, the coefficients of low and high frequency sub-band of the decomposed images are fused separately to obtain a fused coefficient map which has features of both the images. The coefficients are fused based upon certain fusion rules different for low frequency sub-band and high frequency sub-band. The fused image is generated by applying inverse stationary wavelet transform on the fused coefficients map. Many wavelet transform based image fusion method has been presented in the literature which find wavelet transform applicable into many areas like medical diagnosis, image analysis and resolution enhancement [9]-[15]. Finally, Clustering technique is applied on the output of image fusion which classifies the changed and unchanged areas into different clusters. The changed areas belong to one cluster while the unchanged areas belong to the other. There are many clustering techniques in the literature like Fuzzy C means clustering [16]-[17], k means clustering [18] and Nonsubsampled contourlet transform (NSCT) based clustering [19].

This paper proposes new fusion rules for stationary wavelet transform. The coefficients of low frequency sub-band has been fused by applying proposed average based rules while the coefficients of high frequency sub-band are fused by proposed neighborhood mean differencing rule.

The organization of the paper includes four sections. The next section introduces the methodology used for change detection. Third section introduces the datasets and parameters used in the experiment. Results and discussion has been presented in the fourth section. Fifth section presents the conclusion.

\section{PROPOSED ALGORITHM}

Consider two co-registered images $\mathrm{I}_{\mathrm{m} 1}=\left\{I_{m 1}(i, j), 1<i<R, 1<j<C\right\}$ and $\mathrm{I}_{\mathrm{m} 2}=\left\{I_{m 2}(i, j), 1<\right.$ $i<R, 1<j<C\}$ of size $\mathrm{R} \times \mathrm{C}$, i.e., of a scene taken at two different times. The proposed approach involves the three main steps as shown in figure 1. In the first step, Log ratio and Mean ratio operators are applied on two multi-temporal images respectively to generate two source images. In the second steps, both the source images are fused by stationary wavelet transform with proposed fusion rules. In the last step, the difference image is classified into changed and unchanged areas by fuzzy c means clustering algorithm to generate a change map.

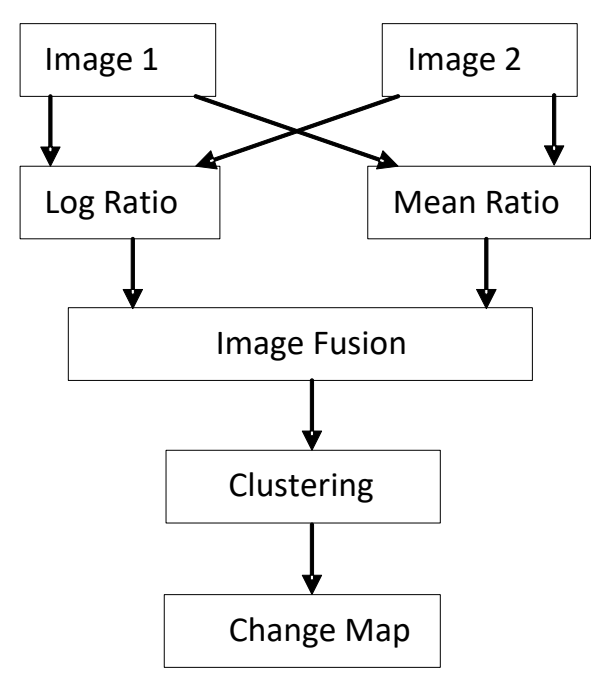

Figure 1. Methodology of proposed change detection approach

In Log Ratio operator, natural logarithms of the ratio of pixels in the images are calculated as given in equation 1.Applying Log operator on an image enhances the low frequency components while suppress the high frequency features in the output image.

$$
I_{l}(i, j)=\log \frac{I_{m 1}(i, j)}{I_{m 2}(i, j)}
$$


In case of Mean Ratio operator, the local mean of the pixel in one image is divided with the local mean of the corresponding pixel in the second image as given in equation 2 .

$$
I_{m}(i, j)=1-\min \left(\frac{\mu_{1}}{\mu_{2}}, \frac{\mu_{2}}{\mu_{1}}\right)
$$

where $\mu_{1}$ and $\mu_{2}$ in equation 2 represents the local mean value of the pixel in the neighborhood of first and second image respectively.

The stationary wavelet transform of each of the source images is computed which gives the wavelet coefficient of both the images. The fusion rules are then applied in order to fuse the corresponding coefficients of the decomposed source images. Different fusion rules are applied on the low frequency and high frequency coefficients. In the next step, inverse stationary level transform (ISWT) is applied on the fused representation to get the fused image.

The first step involved in the process is the decomposition of $\mathrm{I}_{1}$ and $\mathrm{I}_{\mathrm{m}}$ into four images of the equal size. The image corresponding to low frequency sub-band represents the approximate portion of the image while other images corresponding to high frequency sub-band represents the information about the lines and edges in the image which include horizontal, vertical and diagonal components. In SWT, the information of low and high pass channel is applied at every level and two sequences are produced at the next stage. The length of the new sequences will be same as that of the first grouping. In SWT, the zeros are inserted between filter coefficients so as to up-sample the filter.

As the low frequency sub-band and high frequency sub-bands represents different features so it is necessary to fuse them through different fusion rules. Here two fusion rules have been proposed:

Fusion Rule for Low frequency sub-band

$$
\mathrm{I}_{\mathrm{LL}}^{\mathrm{f}}=\alpha \times \max \left(\mathrm{I}_{\mathrm{LL}}^{\mathrm{l}}, \mathrm{I}_{\mathrm{LL}}^{\mathrm{m}}\right)+(1+\alpha) \times \frac{\left(\mathrm{I}_{\mathrm{LL}}^{\mathrm{l}}+\mathrm{I}_{\mathrm{LL}}^{\mathrm{m}}\right)}{2}
$$

Fusion Rule for high frequency Sub-band

$$
\mathrm{I}_{\epsilon}^{\mathrm{f}}=\max \left(\mu_{\epsilon}^{1}, \mu_{\epsilon}^{\mathrm{m}}\right)-\min \left(\mu_{\epsilon}^{\mathrm{l}}, \mu_{\epsilon}^{\mathrm{m}}\right)
$$

Here $\alpha$ in equation 3 is a positive number. $\mathrm{I}^{\mathrm{m}}, \mathrm{I}^{\mathrm{l}}$ and $\mathrm{I}^{\mathrm{f}}$ represents the mean ratio, log ratio and fused images respectively. $\mathrm{I}_{\mathrm{LL}}$ represents the coefficients of low frequency sub-band while $\mathrm{I} \varepsilon(\varepsilon=\mathrm{HL}, \mathrm{LH}$ and $\mathrm{HH})$ represents the coefficients of high frequency sub-band, $\mu_{\epsilon}$ represents the local mean of the coefficients of the neighborhood window in the high frequency sub-band. A window size of $3 \times 3$ has been considered in the algorithm. As the low and high frequency components are obtained by combining the spatial and gray level information of the neighbors, so, the fusion rules further reduces the effect of speckle noise. The proposed rules for low frequency sub-band enhance the edge features of changed regions of the source image while in high frequency sub-band, the fusion rule is selected in such a way to suppress the background information and make the image smoother. So based upon the proposed fusion rules, the change detection output results in maximum background suppression and enhanced features in the changed image.

\section{DATASET AND PARAMETERS}

The image dataset used for calculating the effectiveness of algorithms belongs to the city of Bern, Switzerland captured by European Remote Sensing 2 satellite in the month of April and May 1999 as shown in Figure 2(a) and 2(b). Between the two dates, the Bern city and airport was flooded by Aare River. The ground truth has been shown in Figure $2(\mathrm{c})$. The image size of $(329 \times 329)$ has been used in this experiment. 


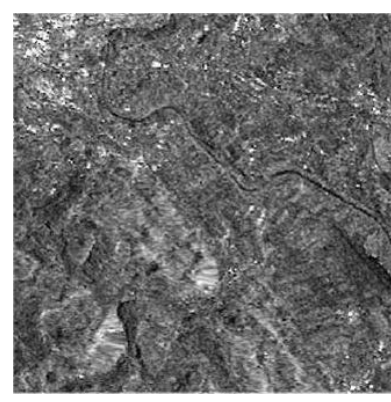

(a)

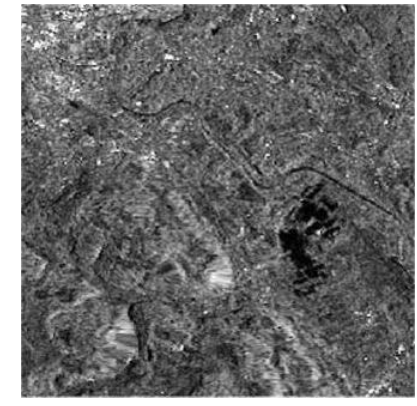

(b)

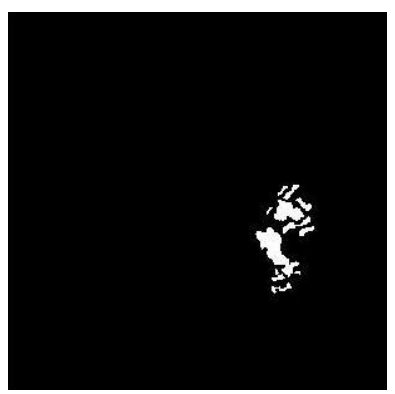

(c)

Figure 2. Multi-temporal images of Bern city (a) Image captured in April 1999 before flooding (b) Image captured in May 1999 after flooding (c) Ground truth [5]

In this paper, the results obtained from proposed method have been compared with the results of log ratio, mean ratio operators, Discrete Wavelet Transform (DWT) based image fusion[5], Neighborhood based ratio approach[20] and Logarithmic ratio based Thresholding[21]. The comparison is done on the basis of various parameters and change image map generated by the algorithms. The parameters used for calculation of effectiveness include percentage correct classification (PCC) and Kappa Coefficient $\left(\mathrm{K}_{\mathrm{c}}\right)$ [22].

$$
\begin{aligned}
& \text { PCC }=\frac{\left(T_{p}+T_{n}\right)}{\left(T_{p}+T_{n}+F_{p}+F_{n}\right)} \\
& \text { If } A=\frac{((T p+F n) x(T p+F p)+(F p+T n) x(T n+F n))}{(T p+T n+F p+T n)^{2}} \\
& K_{c}=\frac{P C C-A}{1-A}
\end{aligned}
$$

$\mathrm{T}_{\mathrm{P}}$ represents the changed pixels which has been identified correctly as changed pixels. The value of $\mathrm{T}_{\mathrm{P}}$ is 1 if the value of corresponding pixels in output of proposed algorithm and ground truth are both 1.Otherwise $T_{P}$ will be zero. $T_{n}$ represents the unchanged pixels which have been correctly identified as unchanged. The value of $\mathrm{T}_{\mathrm{n}}$ is 1 if the corresponding pixels value in output of proposed algorithm and ground truth are both 0 . Otherwise $T_{n}$ will be zero. $F_{P}$ represents those pixels which are actually changed but identified as unchanged pixels. The value of $\mathrm{F}_{\mathrm{P}}$ is 1 if the pixels value in output of algorithm is 1 and the value of corresponding pixel in ground truth is 0 . Otherwise $F_{P}$ will be zero. $F_{n}$ represents those unchanged pixels which have been identified wrongly as changed. The value of $F_{n}$ is 1 if the pixels value in the output of algorithm is 0 and the value of corresponding pixel in ground truth is 1 . Otherwise $F_{n}$ will be zero. Overall Error $(\mathrm{OE})$ is the sum of $\mathrm{F}_{\mathrm{P}}$ and $\mathrm{F}_{\mathrm{n}}$. PCC gives the percentage of pixels correctly identified by the change detection algorithm. $\mathrm{K}_{\mathrm{c}}$ represents the agreement between the output of change detection algorithm with the ground truth.

\section{RESULTS AND DISCUSSION}

To find the effectiveness, the proposed algorithm has been applied on the image dataset of city of Bern. The qualitative analysis has been done by generating the change map as shown in Figure 3. The 
quantitative analysis has been obtained by comparing the output with ground truth through various parameters like false alarms, percentage correct classification, overall error and Kappa coefficient as given in Table 1. The proposed method has yielded highest PCC equal to $99.68 \%$ while PCC for Discrete wavelet transform based image fusion is $99.37 \%$, PCC for neighborhood ratio based method is $99.66 \%$ and PCC for Logarithmic mean based Thresholding is $97.32 \%$. The log ratio operator improves the change detection result by converting the multiplicative speckle noise into additive. So, the output of log ratio operator contains less speckle noise. But, the log ratio operator results in large number of false alarms which means that it is not capable of recovering the maximum unchanged pixels. On the other hand, mean ratio operator results in large amount of speckle noise but it recovers the maximum number of unchanged pixels. The output of both the operators has been fused together to recover maximum number of changed and unchanged pixels. By introducing neighborhood mean for the wavelet coefficients, the change detection has resulted in detection of more changed and unchanged pixels. Also, the neighborhood ratio combines the gray level and spatial information in the neighboring pixels which results in further reduction of speckle noise.

As shown in figure 3(f), the change map generated by proposed method has least spots and maximum resemblance with the ground truth as compare to the other methods used for comparison. The change map generated by log ratio method has less speckle noise but it could not recover the changed and unchanged pixels more effectively. On the other hand, mean ratio has resulted in large number of false alarms which can also be seen in figure 3(b) where large number of white spots exists. Discrete wavelet transform has been effective in recovering the maximum unchanged pixels but it has not been effective in recovering the changed pixels. if the change map of proposed algorithm is compared with the other techniques then it is found that the proposed algorithm has resulted in maximum resemblance with the ground truth. The proposed algorithm has also yielded highest value of Kappa coefficient equal to 0.9958 while it is 0.9952 for Log Ratio, 0.7515 for Mean Ratio, 0.790 for Discrete Wavelet transform, 0.850 for Neighborhood Ratio method and 0.6928 for Logarithmic mean Thresholding based change detection method. This means that the output of proposed method has the most agreement with the ground truth which makes it most accurate among the other methods mentioned in this paper.

Table 1. Results of Change Detection

\begin{tabular}{|c|c|c|c|c|c|c|}
\hline \multirow[b]{2}{*}{ Criterion } & \multicolumn{6}{|c|}{ Method } \\
\hline & Log Ratio & Mean Ratio & $\begin{array}{c}\text { Discrete Wavelet } \\
\text { Transform }\end{array}$ & $\begin{array}{c}\text { Neighborhood } \\
\text { Ratio } \\
\end{array}$ & $\begin{array}{l}\text { Logarithmic Mean } \\
\text { Thresholding }\end{array}$ & Proposed \\
\hline$\overline{F_{p}}$ & 37 & 17115 & 507 & 110 & 677 & 139 \\
\hline$F_{n}$ & 293 & 6 & 61 & 199 & 1077 & 150 \\
\hline $\mathrm{OE}$ & 330 & 17121 & 568 & 309 & 1754 & 289 \\
\hline $\mathrm{PCC}$ & $99.64 \%$ & $81.23 \%$ & $99.37 \%$ & $99.66 \%$ & $97.32 \%$ & $99.68 \%$ \\
\hline Kappa & 0.9952 & 0.7515 & 0.790 & 0.859 & 0.6928 & 0.9958 \\
\hline
\end{tabular}

Figure 3 show the change map of Bern dataset as obtained by log ratio, mean ratio, DWT-FCM, neighborhood ratio approach, logarithmic mean thresholding and proposed algorithm. It can be seen that the change map obtained from the proposed method has recovered the maximum target pixels and reduce the speckle noise.

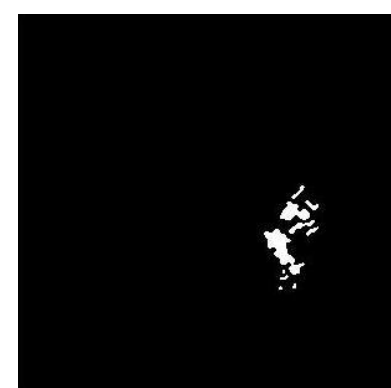

(a)

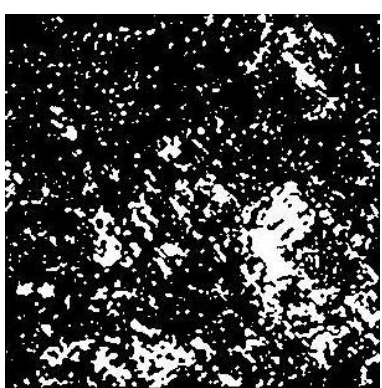

(b)

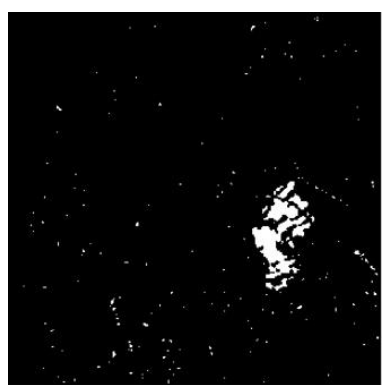

(c) 


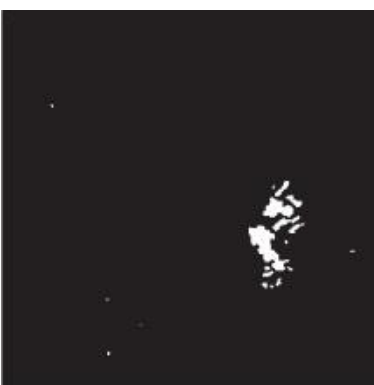

(d)

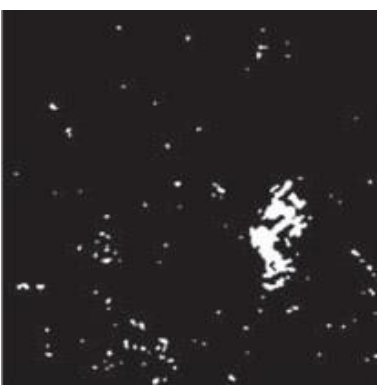

(e)

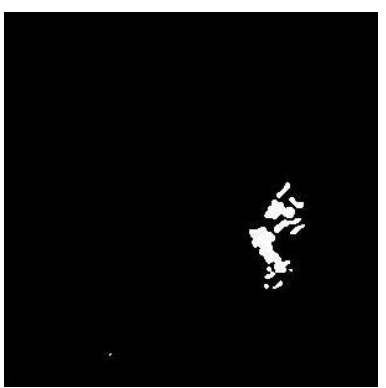

(f)

Figure 3. Change map generated by. (a) Log Ratio (b) Mean Ratio (c) DWT-FCM (d)Neighborhood Ratio approach (e) Logarithmic Mean Thresholding (f)Proposed Method

\section{CONCLUSION}

This paper presented an approach based on Stationary wavelet transform with new fusion rules. The source images generated by $\log$ ratio and mean ratio operator are fused with new set of fusion rules proposed in this paper. The fusion rules for low frequency sub-band enhance the edge features of the changed region. The fusion rule for high frequency sub-band combines the spatial and gray level information of the neighboring pixel which results in reduction of speckle noise from the images. So, the fused image get enhances and contain less amount of speckle noise. The quantitative analysis proves that the accuracy and kappa value of the proposed algorithm is better than its preexistences. As far as qualitative comparison is concerned, the proposed methods offer the least overall error and maximum resemblance with the ground truth. So, based upon the analysis of results it is concluded that the proposed algorithm is more accurate as compare to the other similar methods.

\section{REFERENCES}

[1] R. J. Radke, et al., "Image Change Detection Algorithms: A Systematic Survey," IEEE Transaction on. Image Processing, vol/issue: 14(3), pp. 294-307, 2005.

[2] A Singh, "Digital Change Detection Techniques Using Remotely Sensed Data," International Journal of Remote Sensing, vol/issue: 10(6), pp. 989-1003, 1989.

[3] A. Sharma and T. Gulati, "Review of Change Detection Techniques for Remotely Sensed Images," International Journal of computer Science and Engineering, vol/issue: 5(1), pp. 22-25, 2017.

[4] H. Zhang and X. Cao, "A Way of Image Fusion Based on Wavelet Transform," IEEE 9th International Conference on Mobile Ad-hoc and Sensor Networks, Dalian, pp. 498-501, 2013.

[5] M. Gong, et al., "Change Detection in Synthetic Aperture Radar Images Based on Image Fusion and Fuzzy Clustering," IEEE Transaction on Image Processing, vol/issue: 21(4), pp. 2141-2151, 2012.

[6] M. Beaulieu, et al., "Multi- Spectral Image Resolution Refinement Using Stationary Wavelet Transform," IGARSS 2003, 2003 IEEE International Geoscience and Remote Sensing Symposium, vol. 6, pp. 4032-4034, 2003.

[7] J. E. Fowler, "The Redundant Discrete Wavelet Transform and Additive Noise," IEEE Transactions on Signal Processing Letters, vol. 12, pp. 629-632, 2005.

[8] Saranya G. and S. N. Devi, "Performance Evaluation for Image Fusion Technique in Medical Images Using Spatial and Transform Method," International Conference on Wireless Communications, Signal Processing and Networking, Chennai, pp. 446-450, 2016.

[9] K. K. Kumar, et al., "Resolution Enhancement Using DWT and SWT by Fusion Techniques with Watermarking," IEEE International Conference on Computational Intelligence and Computing Research, Coimbatore, pp. 1-5, 2014.

[10] P. Borwonwatanadelok, et al., "Multi Focus Image Fusion Based on Stationary Wavelet Transform and Extended Spatial Frequency Measurement," IEEE Transaction on Electronic Computer Technology, pp. 77-81, 2009.

[11] F. N. Jamaluddin, et al., "Performance of DWT and SWT in Muscle Fatigue Detection," IEEE Student Symposium in Biomedical Engineering \& Sciences, Shah Alam, pp. 50-53, 2015.

[12] H. Shi and M. Fang, "Multi-focus Color Image Fusion Based on SWT and IHS," Fourth International Conference on Fuzzy Systems and Knowledge Discovery, Haikou, pp. 461-465, 2007.

[13] T. Li and Y. Wang, "Biological Image Fusion Using A SWT Based Variable-Weights Selection Scheme," 3rd International Conference on Bioinformatics and Biomedical Engineering, Beijing, pp. 1-4, 2009.

[14] B. Tian, et al., "Remote Sensing Image Fusion Scheme using Directional Vector in NSCT Domain," TELKOMNIKA Telecommunication, Computing, Electronics and Control, vol/issue: 14(2), pp. 598-606, 2016.

[15] Yuhendra and J. T. S. Sumantyo, "A Quality of Images Fusion for Remote Sensing Application," TELKOMNIKA Telecommunication, Computing, Electronics and Control, vol/issue: 14(1), pp. 378-386, 2016 
[16] J. C. Bezdek, "Pattern Recognition with Fuzzy Objective Function," New York, Plenum, 1981.

[17] J. C. Dunn, "A Fuzzy Relative of the ISODATA Process and Its Use in Detecting Compact Well-Separated Clusters," Journal of Cybernetics, vol. 3, pp. 32-57, 1973.

[18] T. Celik, "Unsupervised Change Detection in Satellite Images Using Principal Component Analysis and k Means Clustering," IEEE Geoscience and Remote Sensing Letters, vol/issue: 6(4), pp. 772-776, 2009.

[19] A. L. da Cunha, et al., "The Nonsubsampled Contourlet Transform: Theory, Design, and Application," IEEE Transactions on Image Processing., vol/issue: 15(10), pp. 3089-3101, 2006.

[20] M. Gong, et al., "A Neighbourhood Based Ratio Approach for Change Detection in SAR Images," IEEE Geoscience and Remote Sensing Letters, vol/issue: 9(2), pp. 307-311, 2012.

[21] M. N. Sumaiya and R. S. S. Kumari, "Logarithmic Mean Based Thresholding for SAR Image Change Detection," IEEE Geoscience and Remote Sensing Letters, vol/issue: 13(11), pp. 1726-1728, 2016.

[22] G. H. Rosenfield and A. F. Lins, "A Coefficient of Agreement as a Measure of Thematic Classification Accuracy," Photogrammetric Engineering and Remote Sensing, vol/issue: 52(2), pp. 223-227, 1986.

\section{BIOGRAPHIES OF AUTHORS}

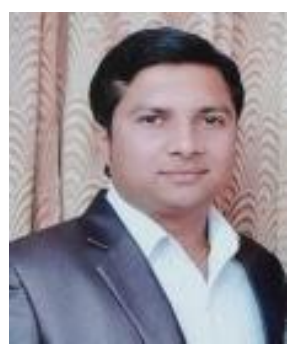

Mr. Abhishek Sharma did Bachelors of Technology from Kurukshetra University, Kurukshetra in 2009 and Master of Technology from Maharishi Markandeshwar University in year 2011. He is currently pursuing Ph.D. in Department of Electronic and Communication, Maharishi Markandeshwar University, Mullana, Ambala. His main research work focuses on change detection in remotely sensed images. He has 8.5 years of teaching and research experience.

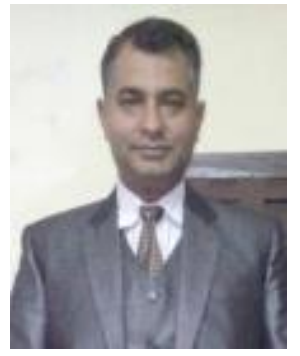

Dr. Tarun Gulati did Bachelors of Technology from Kurukshetra University, Kurukshetra and Master of Technology from NITTTR, Chandigarh. He did Ph.D from M.M. University and currently working as Associate Professor in Department of Electronics \& Communication Engineering, M.M. Engineering College, Maharishi Markandeshwar University, Mullana, Ambala, India. His area of research is image processing and he has 14.5 years of teaching experience. 